\title{
Complete null agent for games with externalities
}

\author{
J.M. Alonso-Meijide ${ }^{1}$, M. Álvarez-Mozos², M.G. Fiestras-Janeiro ${ }^{3}$, and A. \\ Jiménez-Losada ${ }^{4}$ \\ ${ }^{1}$ Dept. de Estatística, Análise Matemática e Optimización, Universidade de Santiago de \\ Compostela, Spain. \\ ${ }^{2}$ Dept. Matemática Econòmica, Financera i Actuarial, Universitat de Barcelona, Spain. \\ ${ }^{3}$ Dept. Estatística e Investigación Operativa, Universidade de Vigo, Spain. \\ ${ }^{4}$ Dept. Matemática Aplicada II, Universidad de Sevilla, Spain.
}

Published in Expert Systems with Applications (2019)

Published version available at http://www.sciencedirect.com/

DOI: $10.1016 /$ j.eswa.2019.05.056

\begin{abstract}
Game theory provides valuable tools to examine expert multi-agent systems. In a cooperative game, collaboration among agents leads to better outcomes. The most important solution for such games is the Shapley value, that coincides with the expected marginal contribution assuming equiprobability. This assumption is not plausible when externalities are present in an expert system. Generalizing the concept of marginal contributions, we propose a new family of Shapley values for situations with externalities. The properties of the Shapley value offer a rationale for its application. This family of values is characterized by extensions of Shapley's axioms: efficiency, additivity, symmetry, and the null player property. The first three axioms have widely accepted generalizations to the framework of games with externalities. However, different concepts of null players have been proposed in the literature and we contribute to this debate with a new one. The null player property that we use is weaker than the others. Finally, we present one particular value of the family, new in the literature, and characterize it by two additional properties.
\end{abstract}

Keywords: game theory; multi-agent systems; externalities; partition function; marginal contribution 


\section{Introduction}

There are many successful applications of game theoretical tools to study expert or intelligent multi-agent problems (see for instance Parsons and Wooldridge, 2002; Pendharkar, 2012). The classic model of games with transferable utility has been thoroughly studied and today it is a theory with solid foundations. It has been widely applied to economic, social, or political problems binding the gap between these fields and mathematics. In particular, it has endowed social sciences with a formal framework in which meaningful statements can be done. One of the main research questions is how to distribute the gains obtained by a given group of agents. In this regard, the Shapley value (Shapley, 1953) is probably the most popular solution and has been used to study a variety of expert systems (Alonso-Meijide and Carreras, 2011; Torkaman et al., 2011). It is defined as the average contribution of a player to its predecessors in a permutation and supported by appealing axiomatic characterizations. The characterizations provide a normative foundation of the value and play an important role in its applications. Most of the contributions in the literature overlook a key fact in today's globally interconnected societies, decisions within a group of agents can affect the outcomes of other groups of agents. Thrall and Lucas (1963) devised the partition function to incorporate coalitional externalities to classic cooperative games. This game theoretical model fits very well to problems where the value of a coalition depends on the actions taken by outsiders. Take for instance the problem of ranking decision making units in data envelopment analysis. Hinojosa et al. (2017) or Li et al. (2018) use cooperative games to obtain a ranking and assume that players outside a coalition always work together. More general situations can be covered by incorporating externalities to these models.

The generalization of the Shapley value to games with externalities has attracted the attention of many scholars. Myerson (1977) was the first to tackle this question by using an axiomatic approach. Some years later, Myerson's value was criticized for not satisfying reasonable monotonicity properties and Bolger (1989) proposed another value by adapting Shapley's original axioms in a different way. Lately, several families of values that generalize the Shapley value to games with externalities have been introduced. Macho-Stadler et al. (2007) used an average approach to build their family, which also contains the value proposed by Albizuri et al. (2005). Dutta et al. (2010) followed the potential approach to define another family that contains the previous one. In Skibski et al. (2018) an even wider family of values is proposed and characterized. All these generalizations are efficient, symmetric, and linear values. Sánchez-Pérez (2015) characterized this wider family of values. Finally, we would like to mention two remarkable generalizations of the Shapley value introduced by de Clippel and Serrano (2008) and McQuillin (2009). Even if the approach and characterization results in the two papers 
are completely independent, the resulting values are in some sense complementary to each other, and they serve us for illustration purposes.

The reason for having so many different generalizations of the Shapley value is the non trivial task of generalizing the concept of the contribution of a player to a coalition. Indeed, when a player leaves a coalition it could either remain alone or join another coalition in the structure. The contribution of a player to a coalition is also the main ingredient of the null player concept. Therefore, from an axiomatic perspective, the way to generalize the null player property is an important difference between the values that can be found in the literature. On the one hand, a weak null player does not affect the worth of any coalition when he leaves it to remain alone. On the other hand, a strong null player does not affect the worth of any coalition by leaving it to either stand alone or join another existing coalition. The properties that correspond to these two notions of null player have been used in the characterization results of Bolger (1989); Macho-Stadler et al. (2007); de Clippel and Serrano (2008); McQuillin (2009), and Skibski et al. (2018), among others. Note that, the null player property is not the only distinguishing property of the existing characterization results which also involve different versions of symmetry, marginality, and consistency properties, for instance.

Here we introduce a new family of values, the so-called lattice structure values (LSvalues), following a different approach to define what the contribution of a player is in a game with externalities. In the classic case, a contribution is based on the movement of a player that joins a coalition and corresponds to a link in the Boolean lattice of subsets. In games with externalities the focus is on embedded coalitions which consist of a coalition and a partition of the complementary coalition. In Alonso-Meijide et al. (2017) we saw that the set of embedded coalitions, $\mathcal{E C}^{N}$, has a (non-Boolean) lattice structure when endowed with the partial order $\sqsubseteq$, defined therein. ${ }^{1}$ Then, we consider that each link in this lattice generates a contribution that could be used to compute a value. However, in this case more than one player could be involved in the change of the embedded coalition. Basically, it could be that a player moves from being alone to joining the active coalition or it could also be that an inactive coalition splits into two new ones. The family of values is parametrized by certain weights that are used to distribute the contributions in which several players are involved. We show that the family of values studied in Skibski et al. (2018) are instances of LS-values which are in turn contained in the class of values studied by Sánchez-Pérez (2015). We deliver an axiomatic characterization of the family of LS-values by means of efficiency, symmetry, linearity, and a new version of the null player property which is weaker than the two

\footnotetext{
${ }^{1}$ Grabisch (2010) was the first to study a partial order over $\mathcal{E C}^{N}$. Using that partial order Grabisch and Funaki (2012) defined several families of values that are not related to ours.
} 
ones described above. Taking into account the way in which we define contributions, we consider that a player is null only if he is never involved in a movement that creates a non-null contribution. We show that if a game with externalities has a player of this type, then the game is actually a classic cooperative game. The basis of the set of games with externalities proposed by de Clippel and Serrano (2008) and the coefficients of any game in this basis - equivalent ot the classic Harsanyi dividends- obtained in AlonsoMeijide et al. (2017) play a relevant role in our results and their exposition. The main difference of our approach with respect to other related contributions is the fact that it is grounded in discrete mathematics rather than in economics. Consequently, some definitions may lack a clear economic interpretation. However, our results provide a way to interpret the key notion of a complete null player. Finally, we single out a particular LS-value, the so-called covering value, that is new in the literature. We also provide a specific characterization of the covering value by means of two proportionality properties.

The rest of the paper is organized as follows. In Section 2 we introduce the model and previous results that we will build upon. Section 3 presents the new family of values and relate it to other remarkable values in the literature. Section 4 is devoted to the axiomatization of the family of values. Before that, we explain what are contributions in this setting and introduce the new null player property. The covering value and its characterization is presented in Section 5. Finally, Section 6 concludes.

\section{Preliminaries}

\subsection{Cooperative games}

A cooperative game with transferable utility is a pair $(N, v)$ where $N$ is a finite set and $v: 2^{N} \rightarrow \mathbb{R}$ is a function with $v(\emptyset)=0$. The elements of $N=\{1,2, \ldots, n\}$ are called players, the subsets $S \subseteq N$ coalitions, $v$ is the characteristic function, and $v(S)$ is the worth of $S$ in the game. For a given $N$ we denote by $G^{N}$ the family of these games with set of players $N$. We may omit the reference to the set of players and only write it explicitly in case it is different from $N$. Player $i \in N$ is a null player in $v \in G^{N}$ if $v(S)=v(S \backslash\{i\})$ for all $S \subseteq N$ with $i \in S$. The unanimity game of coalition $T \subseteq N$, $T \neq \emptyset$, is denoted by $u_{T}$ and defined for every $S \subseteq N$ by $u_{T}(S)=1$, if $T \subseteq S$ and $u_{T}(S)=0$, otherwise. Unanimity games constitute a basis of the vector space $G^{N}$. Indeed, every game $v$ can be written as a linear combination of unanimity games as follows

$$
v=\sum_{T \subseteq N: T \neq \emptyset} \Delta_{T}^{v} u_{T}, \text { with } \Delta_{T}^{v}=\sum_{S \subseteq T}(-1)^{|T|-|S|} v(S) .
$$


The coefficients of the above combination, $\Delta_{T}^{v}$, for all non-empty coalition $T \subseteq N$, are called Harsanyi dividends (Harsanyi, 1963) of the game. They satisfy

$$
\sum_{S \subseteq T} \Delta_{S}^{v}=v(T)
$$

A value on $G^{N}$ assigns to each game $v \in G^{N}$ a payoff vector in $\mathbb{R}^{N}$, where each component represents the payment to a player according to his cooperation possibilities. The Shapley value (Shapley, 1953) of a game $v \in G^{N}$ is defined for any player $i \in N$ by

$$
\phi_{i}(v)=\sum_{S \subseteq N: i \in S} \gamma_{S}[v(S)-v(S \backslash\{i\})],
$$

where $\gamma_{S}=\frac{(|S|-1) !(n-|S|) !}{n !}$. If $T \subseteq N, T \neq \emptyset$, then for each player $i \in N$ we have

$$
\phi_{i}\left(u_{T}\right)= \begin{cases}\frac{1}{|T|}, & \text { if } i \in T \\ 0, & \text { otherwise. }\end{cases}
$$

This value is the only one satisfying the axioms below. A permutation of $N$ is a bijective mapping $\theta: N \longrightarrow N$. Let $\Theta^{N}$ be the set of permutations of $N$. If $\theta \in \Theta^{N}$ then for each $v \in G^{N}$ we define the game $\theta v$ by $\theta v(S)=v\left(\theta^{-1}(S)\right)$ for every $S \subseteq N$, being $\theta^{-1}$ the inverse permutation of $\theta$. Let $f$ be a value on $G^{N}$.

(S1) Linearity. For every $a, b \in \mathbb{R}$ and $v, w \in G^{N}, f(a v+b w)=a f(v)+b f(w)$.

(S2) Efficiency. For every $v \in G^{N}, \sum_{i \in N} f_{i}(v)=v(N)$.

(S3) Symmetry. For every $v \in G^{N}, i \in N$, and $\theta \in \Theta^{N}, f_{\theta(i)}(\theta v)=f_{i}(v)$.

(S4) Null player axiom. If $i \in N$ is a null player in $v \in G^{N}$, then $f_{i}(v)=0$.

These axioms are quite compelling properties. Efficiency states that the value splits the whole gain of the grand coalition. Symmetry says that the value is not affected by the names of the players. Null player property requires that if a player does not contribute to the creation of worth of any coalition, then the value assigns zero to this player. Finally, linearity is a standard technical property.

\subsection{Partitions and embedded coalitions}

Let $\Pi_{N}$ denote the set of partitions of a finite set $N{ }^{2}$ Let $P, Q \in \Pi_{N}$, we say that $P$ is finer than $Q$ and write $P \preceq Q$ if for all $S \in P$ there is $T \in Q$ such that $S \subseteq T$. We write $P \prec Q$ when $P \preceq Q$ but $P \neq Q$. ( $\left.\Pi_{N}, \preceq\right)$ is a lattice. If $P \preceq Q$, then

$$
[P, Q]_{\preceq}=\left\{M \in \Pi_{N}: P \preceq M \preceq Q\right\} .
$$

\footnotetext{
${ }^{2}$ We abuse notation and use $\emptyset$ to denote the only partition in $\Pi_{\emptyset}$.
} 
The top of this poset is $\lceil N\rceil=\{N\}$ and the bottom $\lfloor N\rfloor=\{\{i\}: i \in N\}$. If $P \preceq Q$ and $Q=\left\{S_{1}, \ldots, S_{|Q|}\right\}$ then

$$
\left(\begin{array}{l}
Q \\
P
\end{array}\right)=\prod_{q=1}^{|Q|}\left(m_{q}-1\right) !
$$

where $m_{q}$ is the number of subsets in which $S_{q}$ is divided in $P$.

If $P \in \Pi_{N}$ and $Q \subseteq P$ then $P_{-Q}=P \backslash Q \in \Pi_{N \backslash \cup_{T \in Q} T}$. If $P \in \Pi_{N \backslash T}$ and $Q \in \Pi_{T}$ then $P_{+Q}=P \cup Q \in \Pi_{N}$.

An embedded coalition is a pair $(S ; P)$ where $S \subseteq N$ and $P \in \Pi_{N \backslash S}$, namely a coalition and a partition of the complementary coalition (we call groups to the subsets in $P)$. We denote by $\mathcal{E C}^{N}$ the set of all embedded coalitions of a finite set $N .(S ; P) \in \mathcal{E C}^{N}$ with $S=\emptyset$ and $P \in \Pi_{N}$ is called empty embedded coalition. $\mathcal{E C}_{0}^{N}$ denotes the set of all non-empty embedded coalitions of a finite set $N$.

\subsection{Games with externalities}

A game with externalities is a pair $(N, v)$ consisting of a finite set of players $N$ and a partition function $v: \mathcal{E} \mathcal{C}^{N} \rightarrow \mathbb{R}$, satisfying $v(\emptyset ; P)=0$. Again, we may omit the reference to the player set and only write it explicitly when it is different from $N$. The amount $v(S ; P)$ should be understood as the utility or worth that coalition $S$ obtains when the group structure $P$ (Aumann and Drèze, 1974) emerges in $N \backslash S$. From now on, we name classic games those games defined in Subsection 2.1. We denote by $\mathcal{G}^{N}$ the set of games with externalities with player set $N . \mathcal{G}^{N}$ is a vector space with the sum and scalar product of functions. For every $(T ; Q) \in \mathcal{E C}_{0}^{N}$, the unanimity game of (the embedded coalition) $(T ; Q)$ is defined by ${ }^{3}$

$$
u_{(T ; Q)}(S ; P)= \begin{cases}1, & \text { if } T \subseteq S \text { and } P_{+\lfloor S \backslash T\rfloor} \preceq Q \\ 0, & \text { otherwise, }\end{cases}
$$

for all $(S ; P) \in \mathcal{E C}^{N}$. It is easy to notice that $u_{(S ; P)}$ is a game with negative externalities as other coalitions becoming larger may cause a decrease in the worth of a coalition (Hafalir, 2007) . Note that the name of the above games is chosen deliberately for the parallelism that exists between them and the basis of classic cooperative games. Indeed, for every $T \subseteq N, T \neq \emptyset$ and every $(S ; P) \in \mathcal{E C}^{N}, u_{T}(S)=u_{(T ;[N \backslash T])}(S ; P)$. The set $\left\{u_{(T ; Q)}:(T ; Q) \in \mathcal{E C}_{0}^{N}\right\}$ is a basis of the vector space $\mathcal{G}^{N}$ (de Clippel and Serrano, 2008).

\footnotetext{
${ }^{3}$ The definition of unanimity games also works for any empty embedded coalition, however we omit them because they play no role in our analysis.
} 
A value on $\mathcal{G}^{N}$ is a mapping $f: \mathcal{G}^{N} \rightarrow \mathbb{R}^{N}$. There are several values in the literature that extend the Shapley value to games with externalities (see for instance, Myerson, 1977; Bolger, 1989; Macho-Stadler et al., 2007; de Clippel and Serrano, 2008; McQuillin, 2009; Skibski et al., 2018). All of them satisfy the logical extension of the first three axioms (S1), (S2), and (S3) that we describe below. If $\theta \in \Theta^{N}$, then for each $v \in \mathcal{G}^{N}$ we define the game $\theta v$ as $\theta v(S ; P)=v\left(\theta^{-1} S ; \theta^{-1} P\right)$ for any $(S ; P) \in \mathcal{E C}^{N}$, where $\theta P=\{\theta T: T \in P\}$. Let $f$ be a value on $\mathcal{G}^{N}$.

(SE1) Linearity. For every $a, b \in \mathbb{R}$ and $v, w \in \mathcal{G}^{N}, f(a v+b w)=a f(v)+b f(w)$.

(SE2) Efficiency. For every $v \in \mathcal{G}^{N}, \sum_{i \in N} f_{i}(v)=v(N ; \emptyset)$.

(SE3) Symmetry. For every $v \in \mathcal{G}^{N}, i \in N$, and $\theta \in \Theta^{N}, f_{\theta(i)}(\theta v)=f_{i}(v)$.

Sánchez-Pérez (2015) analyzed and characterized all the values on $\mathcal{G}^{N}$ that satisfy the above axioms. However, the notion of a null player has been extended in several ways and there are different corresponding properties (see for instance, Dutta et al., 2010). We present them explicitly and elaborate on this point in Section 4.

\section{The family of LS-values}

From the definition of a unanimity game with externalities we can infer a binary relation, $\sqsubseteq$, among embedded coalitions. Let $(S ; P),(T ; Q) \in \mathcal{E C}^{N}$, we say that $(T ; Q)$ is contained in $(S ; P)$ and write $(T ; Q) \sqsubseteq(S ; P)$ if and only if $u_{(T ; Q)}(S ; P)=1$. We write $(T ; Q) \sqsubset(S ; P)$ if $(T ; Q) \sqsubseteq(S ; P)$ and $(T ; Q) \neq(S ; P)$. In Alonso-Meijide et al. (2017) the lattice structure of the poset $\left(\mathcal{E C}^{N}, \sqsubseteq\right)$ is thoroughly studied ${ }^{4}$. Figure 1 depicts the Hasse diagram of this poset of embedded coalitions for three players. In that paper, the coefficients of any game in the basis of unanimity games are obtained explicitly. Below, we adjust Proposition 12 in Alonso-Meijide et al. (2017) to the structure with all the embedded coalitions (also the empty ones).

Proposition 3.1. (Alonso-Meijide et al., 2017) If $v \in \mathcal{G}^{N}$ then

$$
v=\sum_{(T ; Q) \in \mathcal{E C}_{0}^{N}} \delta_{(T ; Q)}^{v} u_{(T ; Q)}
$$

where

$$
\delta_{(T ; Q)}^{v}=\sum_{M \in[Q, N \backslash T]_{\preceq}} \sum_{R \subseteq T}(-1)^{|Q|-|M|-|R|}\left(\begin{array}{c}
M \\
Q
\end{array}\right) v\left(T \backslash R ; M_{+\lfloor R\rfloor}\right) .
$$

\footnotetext{
${ }^{4}$ To be precise, in that paper empty embedded coalitions, $(\emptyset ; P)$ with $P \in \Pi(N)$, are not considered. Instead, a fictitious bottom element is added.
} 


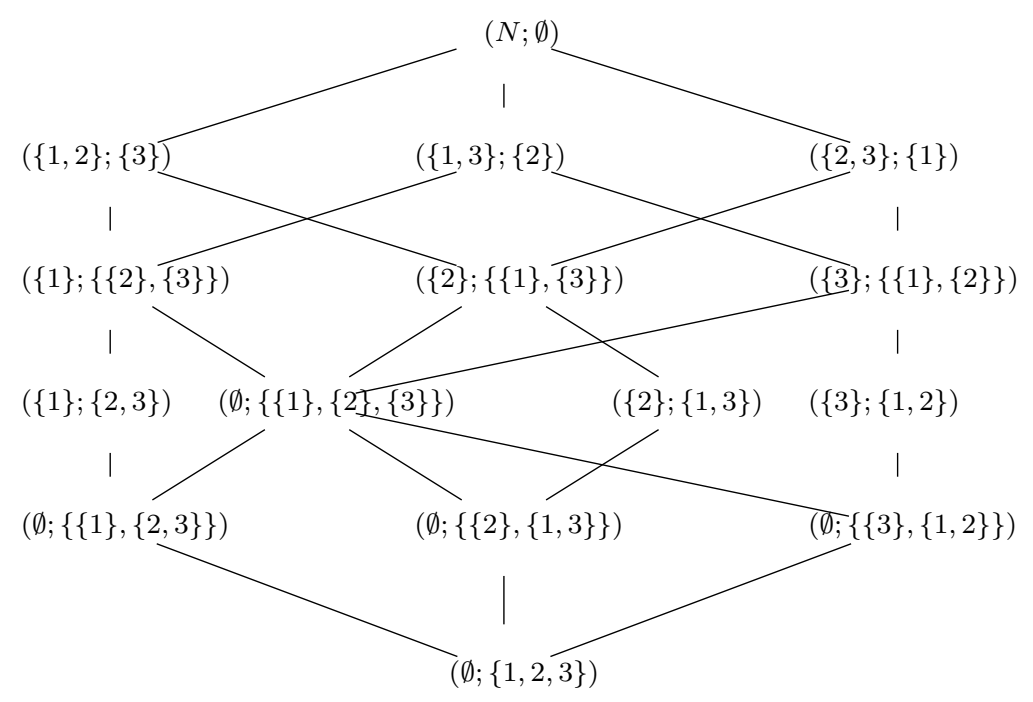

Figure 1: Lattice structure of $\left(\mathcal{E C}^{N}, \sqsubseteq\right)$ with $N=\{1,2,3\}$.

Following Harsanyi (1963), we call dividends to the coefficients $\delta_{(T ; Q)}^{v}$ in the above proposition. These coefficients can also be obtained from a recursive procedure, as it is the case for the dividends of a classic game.

Lemma 3.1. Let $v \in \mathcal{G}^{N}$ be a game with externalities. If $(T ; Q) \in \mathcal{E C}_{0}^{N}$ then

$$
\delta_{(T ; Q)}^{v}=v(T ; Q)-\sum_{\substack{(S ; P) \in \mathcal{E} \mathcal{C}_{0}^{N} \\(S ; P) \sqsubset(T ; Q)}} \delta_{(S ; P)}^{v} .
$$

Proof. From Alonso-Meijide et al. (2017) we know that for each $(T ; Q) \in \mathcal{E C}_{0}^{N}$,

$$
\delta_{(T ; Q)}^{v}=\sum_{\substack{(S ; P) \in \mathcal{E C}_{0}^{N} \\(S ; P) \sqsubseteq(T ; Q)}} \mu((S ; P),(T ; Q)) v(S ; P),
$$

where $\mu$ is the Möbius function of the poset $\left(\mathcal{E C}^{N}, \sqsubseteq\right)$. Hence, the Möbius inversion formula (see for instance Stanley, 2011) means that

$$
v(T ; Q)=\sum_{\substack{(S ; P) \in \mathcal{E} \mathcal{C}_{0}^{N} \\(S ; P) \sqsubseteq(T ; Q)}} \delta_{(S ; P)}^{v}=\delta_{(T ; Q)}^{v}+\sum_{\substack{(S ; P) \in \mathcal{E C}_{0}^{N} \\(S ; P) \sqsubset(T ; Q)}} \delta_{(S ; P)}^{v} .
$$

Assuming linearity as a desirable condition for a value we can introduce a new family of values taking into account Proposition 3.1. We only need to define the outcome for the unanimity games defined in Equation (4). Let $N$ be a finite set with $|N|=n$. 
Definition 3.1. A number with externalities for $n$ is a tuple, $\left(t ; r_{1}^{\lambda_{1}}, \ldots, r_{p}^{\lambda_{p}}\right)$, satisfying 1. $t, r_{1}, \ldots, r_{p}, \lambda_{1}, \ldots, \lambda_{p} \in \mathbb{N}$

2. $r_{1}<\cdots<r_{p}$

3. $t+\sum_{k=1}^{p} \lambda_{k} r_{k}=n$.

The interior of the number is $t$, the externalities are $r_{1}, \ldots, r_{p}$ and their multiplicities are $\lambda_{1}, \ldots, \lambda_{p}$. Besides, $(n ; 0)$ is considered a number with externalities. The set of numbers with externalities for $n$ is denoted by $E_{n}$.

In a number with externalities we do not write the multiplicity if it is one. For instance, if $n=4$ then

$$
E_{4}=\left\{(4 ; 0),(3 ; 1),(2 ; 2),\left(2 ; 1^{2}\right),(1 ; 3),(1 ; 1,2),\left(1 ; 1^{3}\right)\right\}
$$

So, we can define the cardinality of a non-empty embedded coalition using a number with externalities.

Definition 3.2. The cardinality of a non-empty embedded coalition $(T ; Q) \in \mathcal{E C}_{0}^{N}$ is defined by

$$
|T ; Q|=\left(|T| ; r_{1}^{\lambda_{1}}, \ldots, r_{p}^{\lambda_{p}}\right) \in E_{n}
$$

where $Q$ consists of $\lambda_{k}$ groups of cardinality $r_{k}$ for all $k=1, \ldots, p$. In particular, $|N ; \emptyset|=(n ; 0)$.

The next definition introduces the coefficients that will be used to allocate the worth in a unanimity game.

Definition 3.3. A unanimity function over $E_{n}$ is a mapping $\alpha$ satisfying for all $\left(t ; r_{1}^{\lambda}, \ldots, r_{p}^{\lambda_{p}}\right) \in E_{n}$

1. $\left(\alpha_{k}\left(t ; r_{1}^{\lambda_{1}}, \ldots, r_{p}^{\lambda_{p}}\right)\right)_{k=0, \ldots, p} \in \mathbb{R}^{p+1}$,

2. $\alpha_{1}(t ; n-t)=0$ with $t>0$,

3. $\sum_{k=0}^{p} \alpha_{k}\left(t ; r_{1}^{\lambda_{1}}, \ldots, r_{p}^{\lambda_{p}}\right)=1$.

The set of unanimity functions is denoted by $F_{N}$. 
We can now introduce our family of values inspired by Proposition 3.1 and Equation (3). For any $u_{(T ; Q)}$ with $(T ; Q) \in \mathcal{E C}_{0}^{N}$, a family of unanimity coefficients determines the influence of each group depending on the position in $(T ; Q)$. Observe that coalition $T$ needs that $N \backslash T$ sorts out in $Q$ to generate the profit. So, $\alpha_{0}(|T ; Q|)$ can be understood as an importance index on the formation of coalition $T$ and $\alpha_{r}(|T ; Q|)$ an importance index on the formation of each group $R \in Q$ with $|R|=r$. Assuming that cooperation is desirable and that coalitions compete for a limited resource, the worst situation for $T$ is the alliance of all the players in $N \backslash T$, thus it is sensible to assume that $\alpha_{1}(|T ;\lceil N \backslash T\rceil|)=0$. The third is just a normalization condition.

Let $\alpha \in F_{N}$ and $(T ; Q) \in \mathcal{E C}_{0}^{N}$ with $|T ; Q|=\left(t ; r_{1}^{\lambda_{1}}, \ldots, r_{p}^{\lambda_{p}}\right)$. If $i \in T$, we take $k(i)=0$ and $|T ; Q|_{i}=t$. For each $i \in R \in Q$, we take $k(i) \in\{1, \ldots, p\}$ such that $|R|=r_{k(i)}$ and $|T ; Q|_{i}=\lambda_{k(i)} r_{k(i)}$.

Definition 3.4. Let $\alpha \in F_{N}$. The lattice structure value associated with $\alpha, L S^{\alpha}$, is defined as the linear extension of the value defined for unanimity games by

$$
L S_{i}^{\alpha}\left(u_{(T ; Q)}\right)=\frac{\alpha_{k(i)}(|T ; Q|)}{|T ; Q|_{i}} .
$$

for $\operatorname{all}(T ; Q) \in \mathcal{E C}_{0}^{N}$ and $i \in N$. The family of LS-values is the $\operatorname{set}\left\{L S^{\alpha}: \alpha \in F_{N}\right\}$.

Hence, if $v \in \mathcal{G}^{N}$ and $\alpha \in F_{N}$ then $L S^{\alpha}$ is determined by

$$
L S^{\alpha}(v)=\sum_{(T ; Q) \in \mathcal{E} \mathcal{C}_{0}^{N}} \delta_{(T ; Q)}^{v} L S^{\alpha}\left(u_{(T ; Q)}\right) .
$$

These solutions can be seen as the application of the Shapley value to a particular classic cooperative game. The next proposition unveils this relation and provides further insights on unanimity functions.

Proposition 3.2. Let $\alpha \in F_{N}$ be a unanimity function. The LS-value associated with $\alpha$ satisfies for each $(T ; Q) \in \mathcal{E C}_{0}^{N}$ with $|T ; Q|=\left(t ; r_{1}^{\lambda_{1}}, \ldots, r_{p}^{\lambda_{p}}\right)$ that

$$
L S^{\alpha}\left(u_{(T ; Q)}\right)=\sum_{k=0}^{p} \alpha_{k}(|T ; Q|) \phi\left(u_{R_{k}}\right),
$$

with $R_{k}= \begin{cases}T, & \text { if } k=0 \\ \bigcup_{\left\{R \in Q:|R|=r_{k}\right\}} R, & \text { if } k \neq 0 .\end{cases}$

Proof. Let $i \in N$. Notice that $N=\cup_{k=0}^{p} R_{k}$ and $R_{k} \cap R_{l}=\emptyset$, for every $k \neq l$. Thus, there is some $k \in\{0, \ldots, p\}$ such that $i \in R_{k}$. If we consider the classic unanimity game of $R_{k}$ and compute player $i$ 's Shapley value, we obtain

$$
\phi_{i}\left(u_{R_{k}}\right)=\frac{1}{\left|R_{k}\right|} \text {. }
$$


If $k=0, R_{k}=T$ and $\left|R_{k}\right|=|T ; Q|_{i}$. If $k>0$, then $\left|R_{k}\right|=\lambda_{k} r_{k}=|T ; Q|_{i}$. Then,

$$
\sum_{l=0}^{p} \alpha_{l}\left(t ; r_{1}^{\lambda_{1}}, \ldots, r_{p}^{\lambda_{p}}\right) \phi_{i}\left(u_{R_{l}}\right)=\frac{\alpha_{k}\left(t ; r_{1}^{\lambda_{1}}, \ldots, r_{p}^{\lambda_{p}}\right)}{|T ; Q|_{i}}=L S_{i}^{\alpha}\left(u_{(T ; Q)}\right),
$$

concluding the proof.

A game $v \in \mathcal{G}^{N}$ is said to be without externalities if $v(S ; P)=v(S ; Q)$ for all $(S ; P),(S ; Q) \in \mathcal{E C}^{N}$. Each game without externalities $v$ is associated with a classic game $w^{v} \in G^{N}$ in the following way, for all $S \subseteq N$

$$
w^{v}(S)=v(S ;\lceil\backslash S\rceil)
$$

Note that we can consider that classic games are contained in the set of games with externalities. The next proposition states that any LS-value coincides with the Shapley value when the game is without externalities.

Proposition 3.3. Let $v \in \mathcal{G}^{N}$ be a game without externalities. Then, $L S^{\alpha}(v)=\phi\left(w^{v}\right)$, for all $\alpha \in F_{N}$.

Proof. Let $v$ be a game without externalities and $\alpha \in F_{N}$. First, we claim that

$$
\delta_{(T ; Q)}^{v}= \begin{cases}\Delta_{T}^{w^{v}}, & \text { if } Q=\lceil N \backslash T\rceil \\ 0, & \text { otherwise. }\end{cases}
$$

We proceed by a double induction: in the cardinality of $T$ and in the cardinality of $Q$. First, we take the base case defined by $|T|=1$ and $|Q|=1$. If $T=\{i\}$, then

$$
\delta_{(\{i\} ;\lceil N \backslash\{i\}\rceil)}^{v}=v(\{i\} ;\lceil N \backslash\{i\}\rceil)=w^{v}(\{i\})=\Delta_{\{i\}}^{w^{v}} .
$$

Now, let $Q \in \Pi(N \backslash T)$ with $|Q|=2$, that is, $Q=\left\{T_{1}, T_{2}\right\}$. Then,

$$
\delta_{\left(\{i\} ;\left\{T_{1}, T_{2}\right\}\right)}^{v}=v\left(\{i\} ;\left\{T_{1}, T_{2}\right\}\right)-v\left(\{i\} ;\left\lceil T_{1} \cup T_{2}\right\rceil\right)=0 .
$$

Suppose the claim is true for all $(\{i\} ; Q)$ with $1<|Q|<m<n-1$. We prove the equality when $|Q|=m$. Following Lemma 3.1,

$$
\delta_{(\{i\} ; Q)}^{v}=v(\{i\} ; Q)-\sum_{P \succ Q} \delta_{(\{i\} ; P)}^{v}=v(\{i\} ; Q)-v(\{i\} ;\lceil N \backslash\{i\}\rceil)=0 .
$$

Now suppose the claim is true for all $(T ; Q)$ with $1 \leq|T|<t \leq n$ and all $Q \in \Pi_{N \backslash T \text {. We }}$ prove the equality when $|T|=t$ and $|Q|=1$. By Lemma 3.1, the induction hypothesis, and Equation (2)

$$
\delta_{(T ;\lceil N \backslash T\rceil)}^{v}=v(T ;\lceil N \backslash T\rceil)-\sum_{S \subsetneq T} \delta_{(S ;\lceil N \backslash S\rceil)}^{v}=w^{v}(T)-\sum_{S \subsetneq T} \Delta_{S}^{w^{v}}=\Delta_{T}^{w^{v}} .
$$


Suppose the claim is true for all $(T ; Q)$ with $|T|=t$ and $1<|Q|<m<n-1$. Consider $(T ; Q)$ with $|Q|=m$. Again by Lemma 3.1, the induction hypothesis, and Equation (2),

$$
\delta_{(T ; Q)}^{v}=v(T ; Q)-\sum_{(S ; P) \sqsubset(T ; Q): P \neq Q} \delta_{(S ; P)}^{v}=w^{v}(T)-\sum_{S \subseteq T} \Delta_{S}^{w^{v}}=0 .
$$

Thus, we prove the claim.

Second, it remains to show $L S^{\alpha}(v)=\phi(v)$. From Proposition 3.2 and the concept of a unanimity function we get for all $T \subseteq N$,

$$
L S^{\alpha}\left(u_{(T ;\lceil N \backslash T\rceil)}\right)=\phi\left(u_{T}\right),
$$

Finally, using Equations (1), (5), (7), and (8) we have

$$
L S^{\alpha}(v)=\sum_{(T ; Q) \in \mathcal{E} \mathcal{C}_{0}^{N}} \delta_{(T ; Q)}^{v} L S^{\alpha}\left(u_{(T ; Q)}\right)=\sum_{T \subseteq N} \Delta_{T}^{w^{v}} \phi\left(u_{T}\right)=\phi\left(w^{v}\right),
$$

and that concludes the proof.

Next, we show that some of the most well-known values in the literature are instances of LS-values. This is the purpose of the next two examples. In the particular case of the second example, this is far from obvious.

Example 1. The externality-free value of de Clippel and Serrano (2008), $f^{c s}$, is a value on $\mathcal{G}^{N}$ defined as the Shapley value of the classic game $v^{*}$, given by $v^{*}(S)=$ $v(S ;\lfloor N \backslash S\rfloor)$. That is, for every $v \in \mathcal{G}^{N}, f^{c s}(v)=\phi\left(v^{*}\right)$. Obviously this value implies a big loss of information. It is clear that $u_{(T ; Q)}^{*}=u_{T}$ for all $(T ; Q) \in \mathcal{E C}_{0}^{N}$ because

$$
(T ; Q) \sqsubseteq(S ;\lfloor N \backslash S\rfloor) \text { if and only if } T \subseteq S .
$$

So, $f^{c s}\left(u_{(T ; Q)}\right)=\phi\left(u_{T}\right)$ and then $f^{c s}=L S^{\alpha}$ with

$$
\alpha\left(t ; r_{1}^{\lambda_{1}}, \ldots, r_{p}^{\lambda_{p}}\right)=(1,0, \ldots, 0) \quad \forall\left(t ; r_{1}^{\lambda_{1}}, \ldots, r_{p}^{\lambda_{p}}\right) \in E_{n} .
$$

Example 2. McQuillin (2009) defined a value on $\mathcal{G}^{N}$ that can be considered the counterpart of the externality-free value. If de Clippel and Serrano (2008) used the top of the lattice structure $\left(\mathcal{E \mathcal { C } ^ { N }}\right.$, $)$ to define the associated classic game, McQuillin (2009) used the bottom. That is, for every $v \in \mathcal{G}^{N}, f^{q}(v)=\phi\left(v^{* *}\right)$, where $v^{* *} \in G^{N}$ is defined by $v^{* *}(S)=v(S ;\lceil N \backslash S\rceil)$ for all $S \subseteq N$.

Let $(T ; Q) \in \mathcal{E C}_{0}^{N}$ and $u_{(T ; Q)}$ the unanimity game of $(T ; Q)$. First, we claim that

$$
u_{(T ; Q)}^{* *}=\sum_{R \in Q} u_{N \backslash R}-(|Q|-1) u_{N} .
$$


If $S \subsetneq N$ then $(T ; Q) \sqsubseteq(S ;\lceil N \backslash S\rceil)$ if and only if there exists $R_{0} \in Q$ with $N \backslash S \subseteq R_{0}$ (namely, $N \backslash R_{0} \subseteq S$ ). Moreover, as $Q$ is a partition, $R_{0}$ is unique. We obtain

$$
u_{(T ; Q)}^{* *}(S)=1=u_{N \backslash R_{0}}(S)=\sum_{R \in Q} u_{N \backslash R}(S) .
$$

It follows immediately that if $(T ; Q) \nsubseteq(S ;\lceil N \backslash S\rceil)$ then

$$
u_{(T ; Q)}^{* *}(S)=0 .
$$

If $S=N$, clearly $(T ; Q) \sqsubseteq(N ; \emptyset)$ and $N \backslash R \subseteq N$ for any $R \in Q$. Thus,

$$
u_{(T ; Q)}^{* *}(N)=1=\sum_{R \in Q} u_{N \backslash R}(N)-(|Q|-1) u_{N}(N) .
$$

In particular, if $|Q|=1$, then $u_{(T ; Q)}^{* *}=u_{T}$.

Second, we check that $f^{q}$ is also a $L S^{\alpha}$ value. If $i \in T$ then $i \in N \backslash R$ for any $R$ and if $i \in R_{0} \in Q$ then $i \in N \backslash R$ for all $R \in Q \backslash R_{0}$. Using the linearity of the Shapley value, Equation (3), and Equation (9), we get

$$
f_{i}^{q}\left(u_{(T ; Q)}\right)=\left\{\begin{array}{cl}
\sum_{R \in Q} \frac{1}{n-|R|}-\frac{|Q|-1}{n}, & \text { if } i \in T \\
\sum_{R \in Q \backslash R_{0}} \frac{1}{n-|R|}-\frac{|Q|-1}{n}, & \text { if } i \in R_{0} \in Q .
\end{array}\right.
$$

Observe that if $|T ; Q|=\left(t ; r_{1}^{\lambda_{1}}, \ldots, r_{p}^{\lambda_{p}}\right)$ then $|Q|=\sum_{k=1}^{p} \lambda_{k}$. Now we take the following unanimity function. If $\left(t ; r_{1}^{\lambda_{1}}, \ldots, r_{p}^{\lambda_{p}}\right) \in E_{n}$, with $p>1$,

$$
\alpha_{0}\left(t ; r_{1}^{\lambda_{1}}, \ldots, r_{p}^{\lambda_{p}}\right)=t\left[\sum_{k=1}^{p} \frac{\lambda_{k}}{n-r_{k}}-\frac{\sum_{k=1}^{p} \lambda_{k}-1}{n}\right],
$$

and if $k_{0} \in\{1, \ldots, p\}$

$$
\alpha_{k_{0}}\left(t ; r_{1}^{\lambda_{1}}, \ldots, r_{p}^{\lambda_{p}}\right)=\lambda_{k_{0}} r_{k_{0}}\left[\sum_{k=1}^{p} \frac{\lambda_{k}}{n-r_{k}}-\frac{\sum_{k=1}^{p} \lambda_{k}-1}{n}-\frac{1}{n-r_{k_{0}}}\right] .
$$

Also $\alpha(t ; n-t)=(1,0)$. Obviously, we have $f^{q}=L S^{\alpha}$. In order to prove that $\alpha$ is a unanimity function, we only need to check the third condition of Definition 3.3. Suppose $\left(t ; r_{1}^{\lambda_{1}}, \ldots, r_{p}^{\lambda_{p}}\right) \in E_{n}$ with $p>1$. We have

$$
\begin{aligned}
\sum_{k_{0}=0}^{p} \alpha_{k_{0}}\left(t ; r_{1}^{\lambda_{1}}, \ldots, r_{p}^{\lambda_{p}}\right) & =t\left[\sum_{k=1}^{p} \frac{\lambda_{k}}{n-r_{k}}-\frac{\sum_{k=1}^{p} \lambda_{k}-1}{n}\right] \\
& +\sum_{k_{0}=1}^{p} \lambda_{k_{0}} r_{k_{0}}\left[\sum_{k=1}^{p} \frac{\lambda_{k}}{n-r_{k}}-\frac{\sum_{k=1}^{p} \lambda_{k}-1}{n}-\frac{1}{n-r_{k_{0}}}\right] .
\end{aligned}
$$


By the third condition of Definition 1,

$$
\begin{aligned}
\sum_{k_{0}=0}^{p} \alpha_{k_{0}}\left(t ; r_{1}^{\lambda_{1}}, \ldots, r_{p}^{\lambda_{p}}\right) & =t\left[\sum_{k=1}^{p} \frac{\lambda_{k}}{n-r_{k}}-\frac{\sum_{k=1}^{p} \lambda_{k}-1}{n}\right] \\
& +(n-t)\left[\sum_{k=1}^{p} \frac{\lambda_{k}}{n-r_{k}}-\frac{\sum_{k=1}^{p} \lambda_{k}-1}{n}\right]-\sum_{k=1}^{p} \frac{\lambda_{k} r_{k}}{n-r_{k}} \\
& =\sum_{k=1}^{p} \frac{n \lambda_{k}}{n-r_{k}}-\sum_{k=1}^{p} \lambda_{k}+1-\sum_{k=1}^{p} \frac{\lambda_{k} r_{k}}{n-r_{k}}=1 .
\end{aligned}
$$

In the next Example we introduce a generalization of the equal division solution, studied for classic games by van den Brink (2007), to games with externalities.

Example 3. The modified equal division solution is a value on $\mathcal{G}^{N}$ defined by taking

$$
\alpha\left(t ; r_{1}^{\lambda_{1}}, \ldots, r_{p}^{\lambda_{p}}\right)= \begin{cases}\frac{1}{n}\left(t, \lambda_{1} r_{1}, \ldots, \lambda_{p} r_{p}\right), & \text { if } p>1 \\ (1,0), & \text { if } p=1\end{cases}
$$

for all $\left(t ; r_{1}^{\lambda_{1}}, \ldots, r_{p}^{\lambda_{p}}\right) \in E_{n}$. Obviously, $\alpha$ is a unanimity function. Following Definition 4 , the LS-value associated to this unanimity function is given by the linear extension of

$$
f_{i}^{m e}\left(u_{(T ; Q)}\right)= \begin{cases}\frac{1}{n}, & \text { if }|Q|>1 \\ \frac{1}{t}, & \text { if }|Q|=1, i \in T \\ 0, & \text { if }|Q|=1, i \notin T\end{cases}
$$

for every $(T ; Q) \in \mathcal{E C}_{0}^{N}$. On the one hand, $f^{m e}$ shares the unit among all agents when $|Q|>1$. On the other hand, $f^{m e}$ shares the unit only among the players in $T$ when $|Q|=1$.

\section{Axiomatization of the family of LS-values}

We introduce a new concept of contribution for a player in a game with externalities. In the classic theory a contribution can be identified with a link in the Boolean algebra $\left(2^{N}, \subseteq\right)$. Our approach uses the lattice structure (see Figure 1 ) of the set of embedded coalitions, $\left(\mathcal{E C}^{N}, \sqsubseteq\right)$. The links in this lattice can be considered an indivisible step in the formation of an embedded coalition and its associated worth. Links can be of two types: a player joins a coalition or a group is divided in two. Accordingly, there are two types of contributions. Each link represents a cover relation in the lattice. Let $(S ; P),(T ; Q) \in \mathcal{E C}^{N}$ be two embedded coalitions, $(T ; Q)$ covers $(S ; P)$ if one of these facts happens, 
a) there exists $i \in T$ with $S=T \backslash\{i\}$ and $P=Q_{+\lfloor\{i\}\rfloor}$, or

b) $S=T$ and there are two different groups $T_{1}, T_{2} \in Q$ with $P=Q_{-\left\{T_{1}, T_{2}\right\}+\left\lceil T_{1} \cup T_{2}\right\rceil}$

Definition 4.1. Let $v \in \mathcal{G}^{N}$ be a game. The contribution when $(T ; Q)$ covers $(S ; P)$ in $v$ is defined by

$$
v(T ; Q)-v(S ; P)
$$

In the classic theory, the contribution is marginal for a player because each link represents a player joining a coalition. Now, there can be several players involved in a contribution. We consider a player active in a link if his affiliation changes between its two endpoints.

Definition 4.2. Let $(S ; P),(T ; Q) \in \mathcal{E C}^{N}$ be two embedded coalitions such that $(T ; Q)$ covers $(S ; P)$. A player $i \in N$ is active in the link, and it is denoted by $(S ; P) \triangleleft_{i}(T ; Q)$, if $S=T \backslash\{i\}$ or $P=Q_{-\left\{T_{1}, T_{2}\right\}+\left\lceil T_{1} \cup T_{2}\right\rceil}$ with $i \in T_{1} \cup T_{2}$.

The contributions of the first type, are called marginal because each of them is attributed to only one player. They are parallel to the marginal contributions in classic games. But now we also have contributions of a second type that we call external. The concept of null player is strongly connected to the idea of a contribution. Two of the most used definitions of null player are the following (see de Clippel and Serrano, 2008; Macho-Stadler et al., 2007). A player $i$ is a weak null player in $v \in \mathcal{G}^{N}$ if for all $(S ; P) \in \mathcal{E C}^{N}$ with $i \in S$ it holds $v\left(S \backslash\{i\} ; P_{+\lceil\{i\}\rceil}\right)=v(S ; P)$. A player $i$ is a strong null player in $v \in \mathcal{G}^{N}$ if for all $(S ; P) \in \mathcal{E C}^{N}$ with $i \in S$ and $T \in P \cup\{\emptyset\}$ it holds $v\left(S \backslash\{i\} ; P_{-\lceil T\rceil+\lceil T \cup\{i\}\rceil}\right)=v(S ; P)$. Obviously any strong null player is also a weak null player. None of these concepts take into account the external contributions. Now we introduce a new notion of null player using the two types of contributions.

Definition 4.3. Let $v \in \mathcal{G}^{N}$ be a game with externalities. A player $i \in N$ is a complete null player in $v$ if $v(S ; P)=v(T ; Q)$ for every $(S ; P),(T ; Q) \in \mathcal{E C}^{N}$ such that $(T ; Q)$ covers $(S ; P)$ and $(S ; P) \triangleleft_{i}(T ; Q)$.

A complete null player has all his contributions null, namely in all the embedded coalitions the contributions with this player active are null. All the complete null players are also strong null players but not vice-versa. The next example shows a game with externalities that illustrates the previous concepts and has a strong null player who is not a complete null player.

Example 4. Consider the game with externalities over $N=\{1,2,3,4\}$ given by

$v(4 ;\{\{1\},\{2\},\{3\}\})=v(4 ;\{\{1\},\{2,3\}\})=v(4 ;\{\{2\},\{1,3\}\})=v(\{3,4\} ;\{\{1\},\{2\}\})=1$, 
and $v(S ; P)=0$ otherwise. The embedded coalition $(\{3,4\} ;\{\{1\},\{2\}\})$ covers $(\{4\} ;\{\{1\},\{2\},\{3\}\})$. In this case player 3 is the unique active player in the link. Besides, it gives rise to a marginal contribution equal to

$$
v(\{3,4\} ;\{\{1\},\{2\}\})-v(4 ;\{\{1\},\{2\},\{3\}\})=1-1=0 .
$$

The embedded coalition $(\{4\} ;\{\{1\},\{2,3\}\})$ covers $(\{4\} ;\{\{1,2,3\}$. Now, players 1,2 , and 3 are the active players in the link. In this case, the associated external contribution is

$$
v(\{4\} ;\{\{1\},\{2,3\}\})-v(\{4\} ;\{1,2,3\})=1-0=1 .
$$

We check that player 3 is a strong null player. Let $(S ; P) \in \mathcal{E C}^{N}$ with $3 \in S$. It holds that if $(S ; P) \neq(\{3,4\} ;\{\{1\},\{2\}\})$, then $v(S ; P)=v(S \backslash\{3\} ; Q)=0$ where

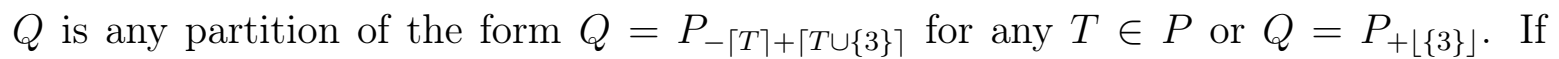
$(S ; P)=(\{3,4\} ;\{\{1\},\{2\}\})$, then

$$
v(S ; P)=v(\{4\} ;\{\{1\},\{2\},\{3\}\})=v(\{4\} ;\{\{1,3\},\{2\}\})=v(\{4\} ;\{\{1\},\{2,3\}\})=1 .
$$

Thus, we prove that player 3 is a strong null player. But player 3 is not a complete null player because

$$
v(\{4\} ;\{\{1\},\{2,3\}\})=1 \text { and } v(\{4\} ;\{1,2,3\})=0 .
$$

Recall that a game without externalities is a game $v \in \mathcal{G}^{N}$ satisfying $v(S ; P)=$ $v(S ; Q)$ for all $(S ; P),(S ; Q) \in \mathcal{E C}^{N}$ and that it can be considered equivalent to a classic one. Indeed, if $v \in \mathcal{G}^{N}$ is a game without externalities then the classic game associated to it (see Equation 6) is defined by $w^{v}(S)=v(S ; P)$ for any $P \in \Pi_{N \backslash S}$. Next, we prove that if there is a complete null player in a game with externalities then the game is equivalent to a classic game.

Proposition 4.1. Let $v \in \mathcal{G}^{N}$. If there exists a complete null player in $v$, then $v$ is a game without externalities. Moreover, this player is a null player in $w^{v} \in G^{N}$.

Proof. Consider that $i \in N$ is a complete null player in the game $v \in \mathcal{G}^{N}$. Let $(S ; P) \in$ $\mathcal{E C}^{N}$ be an embedded coalition. We show that $v(S ; P)=v(S ;\lceil N \backslash S\rceil)$.

Suppose first that $i \notin S$. We proceed by induction on $|P|$. Obviously the result is true when $|P|=1$. If $|P|=2$ then the result follows from the concept of complete null player because if $P=\left\{T_{1}, T_{2}\right\},\left(S ;\left\{T_{1}, T_{2}\right\}\right)$ covers $\left(S ;\left\lceil T_{1} \cup T_{2}\right\rceil\right), i \in T_{1} \cup T_{2}$, and $\left(S ;\left\{T_{1} \cup T_{2}\right\}\right) \triangleleft_{i}\left(S ;\left\{T_{1}, T_{2}\right\}\right)$. Now, assuming that the result is true when $|P|=k-1$ we prove it for $|P|=k$. Let $T_{1}, T_{2} \in P$ with $i \in T_{1} \cup T_{2}$. Then,

$$
v(S ; P)=v\left(S ; P_{-\left\{T_{1}, T_{2}\right\}+\left\lceil T_{1} \cup T_{2}\right\rceil}\right)=v(S ;\lceil N \backslash S\rceil),
$$


where the first equality follows from the definition of complete null player and the second one by the induction hypothesis. Then, $v(S ; P)=v(S ;\lceil N \backslash S\rceil)$.

Secondly, suppose that $i \in S$. The definition of complete null player and the above result imply

$v(S ; P)=v\left(S \backslash\{i\} ; P_{+\lfloor\{i\}\rfloor}\right)=v(S \backslash\{i\} ;\lceil N \backslash S \cup\{i\}\rceil)=v(S \backslash\{i\} ;\{N \backslash S,\{i\}\})=v(S ;\lceil N \backslash S\rceil)$.

Finally, we show that player $i$ is a null player in $w^{v}$. Let $S \subseteq N$ with $i \in S$ and $P \in \Pi_{N \backslash S}$. Then,

$$
w^{v}(S)=v(S ; P)=v\left(S \backslash\{i\} ; P_{+\lfloor\{i\}\rfloor}\right)=w^{v}(S \backslash\{i\})
$$

that is, player $i$ is null player in $w^{v}$ and we conclude the proof.

Sánchez-Pérez (2015) introduced and characterized a family of values by means of linearity (SE1), efficiency (SE2), and symmetry (SE3). We provide the family of LSvalues with an axiomatization using the same axioms (SE1, SE2, and SE3) and a new null player property. Before, we present two existing null player axioms (see for instance de Clippel and Serrano, 2008; Macho-Stadler et al., 2007). Let $f$ be a value on $\mathcal{G}^{N}$. (SE4a) Weak null player property. If $i \in N$ is a weak null player in $v$ then $f_{i}(v)=0$. (SE4b) Strong null player property. If $i \in N$ is a strong null player in $v$ then $f_{i}(v)=0$.

The externality-free value (de Clippel and Serrano, 2008) is the only value on $\mathcal{G}^{N}$ satisfying (SE1), (SE2), (SE3), and (SE4a). Skibski et al. (2018) described the family of values for games with externalities satisfying (SE1), (SE2), (SE3), and (SE4b). Next, we introduce a new property based on the new concept of null player.

(SE4c) Complete null player property. If $i \in N$ is a complete null player in $v$ then $f_{i}(v)=0$.

Let $N$ be a finite set, $v \in \mathcal{G}^{N}$, and $i \in N$. Let $f$ be a value that satisfies (SE4b). If $i$ is a complete null player, then it is also a strong null player. Then, $f_{i}(N, v)=0$ and we conclude that (SE4b) implies (SE4c). Let $g$ be a value that satisfies (SE4a). If $i$ is a strong null player, then it is also a weak null player. Then, $g_{i}(N, v)=0$ and we conclude that (SE4a) implies (SE4b). Summarizing, (SE4a) implies (SE4b) and (SE4b) implies (SE4c).

Next, we show that every LS-value satisfies the axioms.

Theorem 4.1. All LS-values satisfy linearity (SE1), efficiency (SE2), symmetry (SE3), and the complete null player property (SE4c).

Proof. Consider the $L S^{\alpha}$-value with $\alpha \in F_{N}$. We check that it satisfies each axiom. (SE1). It follows directly from Definition 3.4. 
(SE2). Taken a game $v \in \mathcal{G}^{N}$, Lemma 3.1 implies

$$
v(N ; \emptyset)=\sum_{(S ; P) \in \mathcal{E C}_{0}^{N}} \delta_{(S ; P)}^{v} .
$$

Since the linearity of the LS-values and Proposition 3.1 we have

$$
\begin{aligned}
\sum_{i \in N} L S_{i}^{\alpha}(v) & =\sum_{i \in N} \sum_{(T ; Q) \in \mathcal{E} \mathcal{C}_{0}^{N}} \delta_{(T ; Q)}^{v} L S_{i}^{\alpha}\left(u_{(T ; Q)}\right)=\sum_{i \in N} \sum_{(T ; Q) \in \mathcal{E} \mathcal{C}_{0}^{N}} \delta_{(T ; Q)}^{v} \frac{\alpha_{k(i)}(|T ; Q|)}{|T ; Q|_{i}} \\
& =\sum_{(T ; Q) \in \mathcal{E} \mathcal{C}_{0}^{N}} \delta_{(T ; Q)}^{v} \sum_{i \in N} \frac{\alpha_{k(i)}(|T ; Q|)}{|T ; Q|_{i}} .
\end{aligned}
$$

Taking into account the definition of a unanimity function, we obtain for each $(T ; Q) \in$ $\mathcal{E C}_{0}^{N}$ with $|T ; Q|=\left(t ; r_{1}^{\lambda_{1}}, \cdots, r_{p}^{\lambda_{p}}\right)$ and $Q=\left\{T_{1}, \ldots, T_{p}\right\}$

$$
\begin{aligned}
\sum_{i \in N} \frac{\alpha_{k(i)}(|T ; Q|)}{|T ; Q|_{i}} & =\sum_{i \in T} \frac{\alpha_{0}\left(t ; r_{1}^{\lambda_{1}}, \cdots, r_{p}^{\lambda_{p}}\right)}{t}+\sum_{k=1}^{p} \lambda_{k} \sum_{i \in T_{k}} \frac{\alpha_{k(i)}\left(t ; r_{1}^{\lambda_{1}}, \cdots, r_{p}^{\lambda_{p}}\right)}{\lambda_{k} r_{k}} \\
& =\sum_{k=0}^{p} \alpha_{k}\left(t ; r_{1}^{\lambda_{1}}, \cdots, r_{p}^{\lambda_{p}}\right)=1 .
\end{aligned}
$$

(SE3). Consider $\theta \in \Theta^{N}$ and $(T ; Q) \in \mathcal{E C}_{0}^{N}$. Note that $\theta u_{(T ; Q)}=u_{(\theta T ; \theta Q)}$. In fact, $\theta u_{(T ; Q)}(S ; P)=u_{(T ; Q)}\left(\theta^{-1} S ; \theta^{-1} P\right)$ and then $\theta u_{(T ; Q)}(S ; P)=1$ if $(T ; Q) \sqsubseteq\left(\theta^{-1} S ; \theta^{-1} P\right)$, namely $(\theta T ; \theta Q) \sqsubseteq(S ; P)$, and $\theta u_{(T ; Q)}(S ; P)=0$ otherwise. But $|T ; Q|=|\theta T ; \theta Q|$ and so we obtain $L S_{\theta i}^{\alpha}\left(\theta u_{(T ; Q)}\right)=L S_{i}^{\alpha}(v)$ for all $i \in N$. If $v \in \mathcal{G}^{N}$ then for each $(T ; Q) \in \mathcal{E C}_{0}^{N}$ we have $\delta_{(\theta T ; \theta Q)}^{\theta v}=\delta_{(T ; Q)}^{v}$. The equality is true when we take an embedded coalition of the form $(\{i\} ;\lceil N \backslash\{i\}\rceil)$ with $i \in N$ because

$$
\delta_{(\{\theta i\} ;\lceil N \backslash\{\theta i\}\rceil)}^{\theta v}=\theta v(\{\theta i\} ;\lceil N \backslash\{\theta i\}\rceil)=v(\{i\} ;\lceil N \backslash\{i\}\rceil)=\delta_{(\{i\} ;\lceil N \backslash\{i\}\rceil)}^{v} .
$$

From Lemma 3.1 we get the equality by induction for all the embedded coalitions. So, by the linearity of the value,

$$
L S_{\theta i}^{\alpha}(\theta v)=\sum_{(\theta T ; \theta Q) \in \mathcal{E C}_{0}^{N}} \delta_{(\theta T ; \theta Q)}^{\theta v} L S_{\theta i}^{\alpha}\left(u_{(\theta T ; \theta Q)}\right)=\sum_{(T ; Q) \in \mathcal{E} \mathcal{C}_{0}^{N}} \delta_{(T ; Q)}^{v} L S_{i}^{\alpha}\left(u_{(T ; Q)}\right)=L S_{i}^{\alpha}(v) .
$$

(SE4c). Suppose there exists a complete null player $i \in N$ in $v \in \mathcal{G}^{N}$. Proposition 4.1 implies that $v$ is a game without externalities and that player $i$ is a null player in $w^{v}$. As the Shapley value satisfies the null player property, then Proposition 3.3 establishes that $L_{i}^{\alpha}(v)=\phi_{i}\left(w^{v}\right)=0$.

The next theorem shows that the only values satisfying these axioms are LS-values. 
Theorem 4.2. If a value on $\mathcal{G}^{N}$ satisfies linearity (SE1), efficiency (SE2), symmetry (SE3), and complete null player property (SE4c), then it is a LS-value.

Proof. Let $f$ be a value on $\mathcal{G}^{N}$ satisfying the axioms. By linearity we only need to find a unanimity function $\alpha$ such that for all $i \in N$ and $(T ; Q) \in \mathcal{E C}_{0}^{N}$,

$$
f_{i}\left(u_{(T ; Q)}\right)=\frac{\alpha_{k(i)}(|T ; Q|)}{|T ; Q|_{i}} .
$$

Let $\left(t ; r_{1}^{\lambda_{1}}, \ldots, r_{p}^{\lambda_{p}}\right) \in E_{n}$ and $(T ; Q) \in \mathcal{E C}^{N}$ with $|T ; Q|=\left(t ; r_{1}^{\lambda_{1}}, \ldots, r_{p}^{\lambda_{p}}\right)$. We take for every $k=1, \ldots, p$

$$
\alpha_{k}\left(t ; r_{1}^{\lambda_{1}}, \ldots, r_{p}^{\lambda_{p}}\right)=\lambda_{k} r_{k} f_{i}\left(u_{(T ; Q)}\right)
$$

being $i \in T^{\prime} \in Q$ with $\left|T^{\prime}\right|=r_{k}$. Besides, we consider

$$
\alpha_{0}\left(t ; r_{1}^{\lambda_{1}}, \ldots, r_{p}^{\lambda_{p}}\right)=t f_{i}\left(u_{(T ; Q)}\right)
$$

with $i \in T$ and $|T|=t$. Symmetry of $f$ guarantees that $\alpha$ is well defined, because the payoff is the same for all the players in groups with the same size for all the embedded coalitions of a given cardinality. Obviously, the function $\alpha$ satisfies the required equality, thus we focus on checking that $\alpha$ is a unanimity function. By definition $\alpha\left(t ; r_{1}^{\lambda_{1}}, \ldots, r_{p}^{\lambda_{p}}\right) \in \mathbb{R}^{p+1}$ for all $\left(t ; r_{1}^{\lambda_{1}}, \ldots, r_{p}^{\lambda_{p}}\right) \in E_{n}$. Let $(t ; n-t)$ with $t>0$. We have

$$
\alpha_{1}(t ; n-t)=(n-t) f_{i}\left(u_{(T ;\lceil N \backslash T\rceil)}\right),
$$

with $|T|=t$ and $i \notin T$. Since $i$ is a complete null player in $u_{(T ;\lceil N \backslash T])}$ and $f$ satisfies the complete null player property, we obtain $\alpha_{1}(t ; n-t)=0$. Now fixed $\left(t ; r_{1}^{\lambda_{1}}, \ldots, r_{p}^{\lambda_{p}}\right) \in$ $E_{n}$. Let $(T ; Q) \in \mathcal{E C}_{0}^{N}$ with $|T ; Q|=\left(t ; r_{1}^{\lambda_{1}}, \ldots, r_{p}^{\lambda_{p}}\right)$. By efficiency and symmetry of $f$, we have

$$
\sum_{k=0}^{p} \alpha_{k}\left(t ; r_{1}^{\lambda_{1}}, \ldots, r_{p}^{\lambda_{p}}\right)=\sum_{k=0}^{p} \lambda_{k} r_{k} f_{i}\left(u_{(T ; Q)}\right)=\sum_{i \in N} f_{i}\left(u_{(T ; Q)}\right)=u_{(T ; Q)}(N ; \emptyset)=1 .
$$

Thus, we finish the proof.

Notice that Equation (10) provides a method to obtain the unanimity function associated to a LS-value from the payoffs in unanimity games.

Sánchez-Pérez (2015) described the family of all values on $\mathcal{G}^{N}$ that satisfy (SE1), (SE2), and (SE3). Thus, the LS-values are instances of that family, but not all the values satisfying these three axioms satisfy the complete null player property. For instance, the equal division value, defined by

$$
f_{i}(v)=\frac{v(N ; \emptyset)}{n},
$$


for all $i \in N$ and $v \in \mathcal{G}^{N}$ satisfies (SE1), (SE2), and (SE3) but not (SE4c). Since (SE4b) implies (SE4c), then all the values on $\mathcal{G}^{N}$ studied by Skibski et al. (2018) are LS-values. But there exist LS-values which do not belong to that family as we see in the next Section.

\section{The covering value}

In this section we introduce a particular LS-value following an interpretation of the Shapley value in classic unanimity games (see Equation (3)). Consider a classic unanimity game $u_{T}$ for a non-empty coalition $T \subseteq N$. The Shapley value shares the unit of worth equally among the contributions to this coalition. We have $|T|$ contributions, in each of them the contribution is marginal for a different player of $T$. Hence, the payoff to a player is the probability of being an active player in a contribution. We extend this idea to unanimity games with externalities, taking into account our considerations on what a contribution for a player is. First, we calculate the number of contributions to a particular embedded coalition.

Proposition 5.1. Let $(T ; Q) \in \mathcal{E C}_{0}^{N}$. The number of embedded coalitions covered by $(T ; Q)$ is

$$
\operatorname{cov}(T ; Q)=|T|+\frac{|Q|(|Q|-1)}{2} .
$$

Proof. There is one for each player in coalition $T$ of the form $\left(T \backslash\{i\} ; Q_{+\lfloor\{i\}\rfloor}\right)$. Furthermore, we have one for each union of two different groups of $Q,\left(T ; Q_{-\{S, R\}+\lceil S \cup R\rceil}\right)$, and there are as many as $\frac{|Q|(|Q|-1)}{2}$ of them.

According to Proposition 5.1, if we take $(T ; Q) \in \mathcal{E C}_{0}^{N}$ with $|Q|=1$, then $\operatorname{cov}(T ; Q)=$ $|T|$.

Take an embedded coalition $(S ; P)$ covered by $(T ; Q)$. If $(S ; P)$ produces a marginal contribution, namely $S=T \backslash\{i\}$ for certain $i \in T$, then we allocate the contribution to player $i$. If $(S ; P)$ generates an external contribution, namely $S=T$, then we share the contribution among the active players in the link, all of them out of $T$, and the players in the coalition $T$. We include players in $T$ because they form the coalition that generates the worth. So, we assign to each player in $T$ a marginal contribution and a part of each external contribution, and we assign to a player not in $T$ a part of those external contributions in which he is active in the link. 
Definition 5.1. The covering value is the linear extension of the function defined by

$$
\kappa_{i}\left(u_{(T ; Q)}\right)= \begin{cases}\frac{1}{\operatorname{cov}(T ; Q)}\left[1+\sum_{R \in U(Q)} \frac{1}{|T|+|R|}\right], & \text { if } i \in T \\ \frac{1}{\operatorname{cov}(T ; Q)} \sum_{\substack{R \in U(Q) \\ i \in R}} \frac{1}{|T|+|R|}, & \text { if } i \notin T\end{cases}
$$

for every $(T ; Q) \in \mathcal{E C}_{0}^{N}$, where $U(Q)=\left\{S \cup S^{\prime}: S, S^{\prime} \in Q, S \neq S^{\prime}\right\}$.

Next we show that the covering value is an instance of the family of LS-values.

Proposition 5.2. The covering value is a LS-value.

Proof. Let $\left(t ; r_{1}^{\lambda_{1}}, \ldots, r_{p}^{\lambda_{p}}\right) \in E_{n}$. We define

$$
\begin{gathered}
\operatorname{cov}\left(t ; r_{1}^{\lambda_{1}}, \ldots, r_{p}^{\lambda_{p}}\right)=t+\frac{\left(\lambda_{1}+\cdots+\lambda_{p}\right)^{2}-\left(\lambda_{1}+\cdots+\lambda_{p}\right)}{2}, \\
\alpha_{0}\left(t ; r_{1}^{\lambda_{1}}, \ldots, r_{p}^{\lambda_{p}}\right)=\frac{t}{\operatorname{cov}\left(t ; r_{1}^{\lambda_{1}}, \ldots, r_{p}^{\lambda_{p}}\right)}\left[1+\sum_{k=1}^{p}\left[\frac{\lambda_{k}^{2}-\lambda_{k}}{2 t+4 r_{k}}+\sum_{m=k+1}^{p} \frac{\lambda_{k} \lambda_{m}}{t+r_{k}+r_{m}}\right]\right],
\end{gathered}
$$

and for all $k=1, \ldots, p$

$$
\alpha_{k}\left(t ; r_{1}^{\lambda_{1}}, \ldots, r_{p}^{\lambda_{p}}\right)=\frac{\lambda_{k} r_{k}}{\operatorname{cov}\left(t ; r_{1}^{\lambda_{1}}, \ldots, r_{p}^{\lambda_{p}}\right)}\left[\frac{\lambda_{k}-1}{t+2 r_{k}}+\sum_{\substack{m=1 \\ m \neq k}}^{p} \frac{\lambda_{m}}{t+r_{k}+r_{m}}\right] .
$$

We have to prove that the covering value can be obtained applying Equation (10) through function $\alpha$ and that $\alpha$ is a unanimity function. First, let $(T ; Q) \in \mathcal{E C}_{0}^{N}$ with $|T ; Q|=\left(t ; r_{1}^{\lambda_{1}}, \ldots, r_{p}^{\lambda_{p}}\right)$. Since $|Q|=\lambda_{1}+\cdots+\lambda_{p}$, then $\operatorname{cov}(T ; Q)=\operatorname{cov}(|T ; Q|)$. Let $i \in T$. We get

$$
\sum_{R \in U(Q)} \frac{1}{|T|+|R|}=\sum_{k=1}^{p}\left[\frac{\lambda_{k}^{2}-\lambda_{k}}{2 t+4 r_{k}}+\sum_{m=k+1}^{p} \frac{\lambda_{k} \lambda_{m}}{t+r_{k}+r_{m}}\right]
$$

by reordering the elements in $U(Q)$ according to the size of the groups in $Q$. Let $i \notin T$. Then, in a similar way we obtain

$$
\sum_{\substack{R \in U(Q) \\ i \in R}} \frac{1}{|T|+|R|}=\frac{\lambda_{k}-1}{t+2 r_{k}}+\sum_{\substack{m=1 \\ m \neq k}}^{p} \frac{\lambda_{m}}{t+r_{k}+r_{m}} .
$$


Combining Equations (11)-(15), we have that

$$
\kappa_{i}\left(u_{(T ; Q)}\right)=\frac{\alpha_{k}(|T ; Q|)}{|T ; Q|_{i}}, \quad \forall k=0, \ldots, p .
$$

It remains to show that $\alpha$ is a unanimity function. By definition $\alpha\left(t ; r_{1}^{\lambda_{1}}, \ldots, r_{p}^{\lambda_{p}}\right) \in$ $\mathbb{R}^{p+1}$. If $t>0$ then $\operatorname{cov}(t ; n-t)=t+0=t$ and

$$
\alpha_{1}(t ; n-t)=\frac{n-t}{t} 0=0
$$

Finally, we need to prove that

$$
\sum_{k=0}^{p} \alpha_{k}\left(t ; r_{1}^{\lambda_{1}}, \ldots, r_{p}^{\lambda_{p}}\right)=1
$$

Observe that

$$
\sum_{k=1}^{p} \frac{t\left(\lambda_{k}^{2}-\lambda_{k}\right)}{2 t+4 r_{k}}+\sum_{k=1}^{p} \frac{r_{k} \lambda_{k}\left(\lambda_{k}-1\right)}{t+2 r_{k}}=\sum_{k=1}^{p} \frac{\lambda_{k}^{2}-\lambda_{k}}{2}
$$

and also

$$
\sum_{k=1}^{p} \sum_{m=k+1}^{p} t \frac{\lambda_{k} \lambda_{m}}{t+r_{k}+r_{m}}+\sum_{k=1}^{p} \lambda_{k} r_{k} \sum_{\substack{m=1 \\ m \neq k}}^{p} \frac{\lambda_{m}}{t+r_{k}+r_{m}}=\sum_{k=1}^{p} \sum_{m=k+1}^{p} \lambda_{k} \lambda_{m} .
$$

Thus, adding up $t$ and Equations (16) and (17), we obtain

$t+\sum_{k=1}^{p} \frac{\lambda_{k}^{2}-\lambda_{k}}{2}+\sum_{k=1}^{p} \sum_{m=k+1}^{p} \lambda_{k} \lambda_{m}=t+\frac{\left(\lambda_{1}+\cdots+\lambda_{p}\right)^{2}-\left(\lambda_{1}+\cdots+\lambda_{p}\right)}{2}=\operatorname{cov}\left(t ; r_{1}^{\lambda_{1}}, \ldots, r_{p}^{\lambda_{p}}\right)$,

which completes the proof.

The covering value is an example of LS-value, then it satisfies (SE4c), but does not satisfy strong null player property. We show this fact in the next example.

Example 5. Let us take up again the game with externalities defined in Example 4. Recall that player 3 is a strong null player in $v$ but he is not a complete null player. We determine player 3's payoff given by the covering value. The game $v$, following Proposition 3.1, can be written as

$v=u_{(\{4\} ;\{1\},\{2,3\})}+u_{(\{4\} ;\{2\},\{1,3\})}-u_{(\{4\} ;\{1\},\{2\},\{3\})}-u_{(\{1,4\} ;\{2,3\})}-u_{(\{2,4\} ;\{1,3\})}+u_{(\{1,2,4\} ;\{3\})}$.

Applying the covering value to the involved unanimity games, we get

$$
\begin{aligned}
& \kappa_{3}\left(u_{(\{4\} ;\{1\},\{2,3\})}\right)=\frac{1}{8} \quad \kappa_{3}\left(u_{(\{4\} ;\{2\},\{1,3\})}\right)=\frac{1}{8} \quad \kappa_{3}\left(u_{(\{4\} ;\{1\},\{2\},\{3\})}\right)=\frac{1}{6} \\
& \kappa_{3}\left(u_{(\{1,4\} ;\{2,3\})}\right)=\kappa_{3}\left(u_{(\{2,4\} ;\{1,3\})}\right)=\kappa_{3}\left(u_{(\{1,2,4\} ;\{3\})}\right)=0 .
\end{aligned}
$$


As $\kappa_{3}(v)=\frac{1}{12} \neq 0$, the covering value does not satisfy the strong null player property. The covering value for all the players is

$$
\kappa(v)=\left(-\frac{1}{12},-\frac{1}{12}, \frac{1}{12}, \frac{1}{12}\right) .
$$

For illustrative purposes, we compute the payoff vectors given by $f^{c s}$ and $f^{q}$, defined in Example 1 and Example 2, respectively. Notice that

$$
v^{*}=u_{\{4\}}-u_{\{1,4\}}-u_{\{2,4\}}+u_{\{1,2,4\}} \text { and } v^{* *}=0 .
$$

Thus,

$$
f^{c s}(v)=\phi\left(v^{*}\right)=\left(-\frac{1}{6},-\frac{1}{6}, 0, \frac{1}{3}\right) \quad \text { and } \quad f^{q}(v)=\phi\left(v^{* *}\right)=(0,0,0,0) .
$$

Proposition 5.2 guarantees that the covering value satisfies linearity (SE1), efficiency (SE2), symmetry (SE3), and complete null player (SE4c). We propose now properties that pin down the covering value. We follow the idea of hierarchical power of Faigle and Kern (1992) in the sense that we propose certain proportionality between the payoffs in a unanimity game and the measure of power for an embedded coalition. Let $(T ; Q) \in \mathcal{E C}_{0}^{N}$ be an embedded coalition. The power in this embedded coalition is measured by two levels: the activity level of coalition $T$ with respect to $Q$

$$
\operatorname{act}^{(T ; Q)}=|T|+\sum_{R \in U(Q)} \frac{|T|}{|T|+|R|}
$$

and the splitting level of $(T ; Q)$

$$
s p l^{(T ; Q)}=\sum_{R \in U(Q)} \frac{|R|}{|T|+|R|} .
$$

The splitting level can be divided among the groups of the partition as follows. For each $S \in Q$,

$$
\operatorname{spl}_{S}^{(T ; Q)}=\sum_{\substack{R \in U(Q) \\ S \subseteq R}} \frac{|S|}{|T|+|R|} .
$$

Observe that $s p l^{(T ; Q)}=0$ if and only if $|Q|=1$. Additionally,

$$
\sum_{S \in Q} s p l_{S}^{(T ; Q)}=s p l^{(T ; Q)} \quad \text { and } \quad a c t^{(T ; Q)}+s p l^{(T ; Q)}=\operatorname{cov}(T ; Q) .
$$

In the following we propose two new properties based on these measures in order to characterize the covering value. Let $f$ be a value on $\mathcal{G}^{N}$. 
(SE5) Proportionality on activity versus splitting. For every embedded coalition $(T ; Q) \in$ $\mathcal{E C}_{0}^{N}$,

$$
s p l^{(T ; Q)} \sum_{i \in T} f_{i}\left(u_{(T ; Q)}\right)=a c t^{(T ; Q)} \sum_{i \in N \backslash T} f_{i}\left(u_{(T ; Q)}\right) .
$$

(SE6) Partial splitting level property. For every embedded coalition $(T ; Q) \in \mathcal{E C}_{0}^{N}$, $S, S^{\prime} \in Q$,

$$
s p l_{S}^{(T ; Q)} \sum_{i \in S^{\prime}} f_{i}\left(u_{(T ; Q)}\right)=s p l_{S^{\prime}}^{(T ; Q)} \sum_{i \in S} f_{i}\left(u_{(T ; Q)}\right) .
$$

To conclude this Section, we characterize the covering value in the next result.

Theorem 5.1. The covering value $\kappa$ is the only value on $\mathcal{G}^{N}$ satisfying linearity (SE1), efficiency (SE2), symmetry (SE3), proportionality on activity versus splitting (SE5), and the partial splitting property (SE6).

Proof. Existence. In Theorem 6, we proved that all LS-values satisfy (SE1), (SE2) and (SE3). It is easy to check that the covering value $\kappa$ satisfies (SE5) and (SE6).

Uniqueness. Let $f$ be a value on $\mathcal{G}^{N}$ satisfying all the axioms in the statement. By (SE1) we only need to show uniqueness for unanimity games. Let $(T ; Q) \in \mathcal{E C}_{0}^{N}$. Since $f$ satisfies (SE2) and (SE5), we obtain

$$
s p l^{(T ; Q)} \sum_{i \in T} f_{i}\left(u_{(T ; Q)}\right)=a c t^{(T ; Q)}\left[1-\sum_{i \in T} f_{i}\left(u_{(T ; Q)}\right)\right] .
$$

Hence,

$$
\sum_{i \in T} f_{i}\left(u_{(T ; Q)}\right)=\frac{a c t^{(T ; Q)}}{a c t^{(T ; Q)}+\operatorname{spl}^{(T ; Q)}} .
$$

Using (SE3), we get $f_{i}\left(u_{(T ; Q)}\right)=f_{j}\left(u_{(T ; Q)}\right)$ for all $i, j \in T$. Then, for each $i \in T$ we have

$$
f_{i}\left(u_{(T ; Q)}\right)=\frac{a c t^{(T ; Q)}}{|T|\left(a c t^{(T ; Q)}+\operatorname{spl}^{(T ; Q)}\right)} .
$$

Applying again (SE3), $f_{i}\left(u_{(T ; Q)}\right)=f_{j}\left(u_{(T ; Q)}\right)$, for all $i, j \in S$ with $S \in Q$. Let $i \notin T$. If $|Q|=1$, namely $Q=\lceil N \backslash T\rceil$, then (SE5) and (SE3) imply

$$
\operatorname{act}^{(T ; Q)}|N \backslash T| f_{i}\left(u_{(T ; Q)}\right)=0,
$$

because the splitting level is $s p l^{(T ; Q)}=0$. Thus, $f_{i}\left(u_{(T ; Q)}\right)=0$. Suppose now that $|Q|>1$. Let us take $S \in Q$ with $i \in S$. Applying (SE6) we have for each $S^{\prime} \in Q$, $S^{\prime} \neq S$,

$$
\sum_{j \in S^{\prime}} f_{j}\left(u_{(T ; Q)}\right)=\frac{s p l_{S^{\prime}}^{(T ; Q)}}{s p l_{S}^{(T ; Q)}} \sum_{j \in S} f_{j}\left(u_{(T ; Q)}\right) .
$$


Using (SE2), we obtain

$$
\begin{aligned}
u_{(T ; Q)}(N ; \emptyset)=1 & =\sum_{j \in T} f_{j}\left(u_{(T ; Q)}\right)+\sum_{S^{\prime} \in Q} \sum_{j \in S^{\prime}} f_{j}\left(u_{(T ; Q)}\right) \\
& =\sum_{j \in T} f_{j}\left(u_{(T ; Q)}\right)+|S| f_{i}\left(u_{(T ; Q)}\right) \sum_{S^{\prime} \in Q} \frac{s p l_{S^{\prime}}^{(T ; Q)}}{s p l_{S}^{(T ; Q)}} \\
& =\sum_{j \in T} f_{j}\left(u_{(T ; Q)}\right)+\frac{|S| s p l^{(T ; Q)}}{s p l_{S}^{(T ; Q)}} f_{i}\left(u_{(T ; Q)}\right) .
\end{aligned}
$$

But, since $f_{j}\left(u_{(T ; Q)}\right)$ is unique for all $j \in T$, then $f_{i}\left(u_{(T ; Q)}\right)$ is also unique, which finishes the proof.

In Theorem 6 we provide a characterization of the family of LS-values, using among others, the complete null player property (SE4c). Even if the covering value is an element of this family, this property is not directly included in Theorem 10. Nevertheless, notice that under symmetry, the definition of the splitting level of an embedded coalition and (SE5) imply the complete null player property (SE4c) as you can see in the proof of Theorem 10 (Equation (18)).

\section{Conclusions}

Inspired by our previous work on a lattice structure of $\mathcal{E C ^ { N }}$, we have built the family of LS-values which contain many of the existing generalizations of the Shapley value. As it is a broad family, it is not easy to find a closed expression for an arbitrary member. The computation in a particular game is also not a simple task as one first needs to write the game as a combination of unanimity games. However, the family of LS-values is supported by an appealing axiomatization that uses four properties that generalize the ones used by Shapley (1953) in his seminal characterization. The first three properties, efficiency, symmetry, and linearity are very standard and have been extensively used in the framework of games with externalities. The fourth one, is the main novelty of the characterization result as it is a new version of the null player property. There is quite a debate about how to generalize the classic property and we contribute to it by introducing a property which is weaker than others. Actually, it can be considered the weakest possible generalization of the classic null player property by the result in Proposition 4.1. Figure 2 depicts the inclusion relation among several families of values for games with externalities. We use boldface letters for families of values. This means that any value in the Skibski et al. (2018) family is a LS-value and any LS-value is a 
value of the Sánchez-Pérez (2015) family. We have just identified a value that is a LSvalue, but that does not belong to the family of Skibski et al. (2018). It is the covering value. Similarly, using the equal division value we have seen that not all values in the Sánchez-Pérez (2015) family are LS-values.

We devise the following future research lines. First, to facilitate the application of values to real examples related to expert systems, methods to ease the computation of values should be developed. Second, we plan to study monotonicity or marginality properties that LS-values might satisfy and explore alternative characterizations of the family. Finally, there are some open questions about how to write other values proposed in the literature, like the ones introduced in Albizuri et al. (2005) or Hu and Yang (2010), as LS-values.

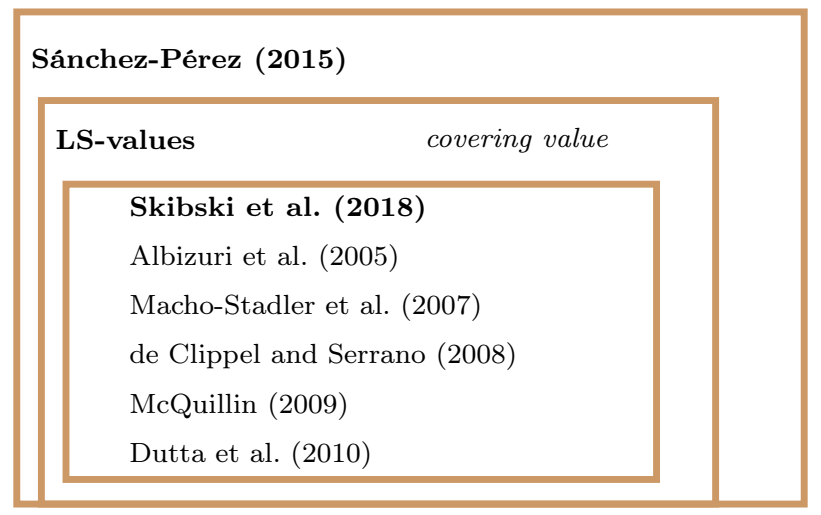

Figure 2: Families of values diagram.

\section{Acknowledgments}

This work has been supported by the European Regional Development Fund (ERDF) and Ministerio de Ciencia, Innovación y Universidades through grants ECO2017-86481P, MTM2017-83455-P, MTM2017-87197-C3-2-P, MTM2017-87197-C3-3-P, by the Generalitat de Catalunya through grant 2017-SGR-778, by the Junta de Andalucía through grant FQM237, and by the Xunta de Galicia through the European Regional Development Fund (Grupos de Referencia Competitiva ED431C-2016-040 and ED431C$2017 / 38)$. 


\section{References}

Albizuri, M.J., Arin, J., and Rubio, J. (2005). An axiom system for a value for games in partition function form. International Game Theory Review 7, 63-72.

Alonso-Meijide J.M., Álvarez-Mozos M., Fiestras-Janeiro G., and Jiménez-Losada A. (2017). Some structural properties of a lattice of embedded coalitions. International Journal of General Systems 46, 123-143.

Alonso-Meijide J.M. and Carreras F. (2011). The proportional coalitional Shapley value. Expert Systems with Applications 38, 6967-6979.

Aumann R.J., and Drèze J.H. (1974). Cooperative games with coalition structures. International Journal of Game Theory 3, 217-237.

van den Brink R. (2007). Null or nullifying players: The difference between the Shapley value and equal division solutions. Journal of Economic Theory 136, 767-775.

Bolger E.M. (1989). A set of axioms for a value for partition function games. International Journal of Game Theory 18, 37-44.

de Clippel G. and Serrano R. (2008). Marginal contributions and externalities in the value. Econometrica 76, 1413-1436.

Dutta B., Ehlers L., and Kar A. (2010). Externalities, potential, value and consistency. Journal of Economic Theory 145, 2380-2411.

Faigle U. and Kern W. (1992). The Shapley value for cooperative games under precedence constraints. International Journal of Game Theory 21, 249-266.

Grabisch, M. (2010). The lattice of embedded subsets. Discrete Applied Mathematics $158,479-488$.

Grabisch, M. and Funaki, Y. (2012). A coalition formation value for games in partition function form. European Journal of Operational Research 221, 175-185.

Hafalir I. (2007). Efficiency in coalition games with externalities. Games and Economic Behavior 61, 242-258.

Harsanyi J.C. (1963). A simplified bargaining model for the $n$-person cooperative game. International Economic Review 4, 194-220. 
Hinojosa M.A., Lozano S., Barrero D.V. and Mármol A.M. (2017). Ranking efficient DMUs using cooperative game theory. Expert Systems with Applications 80, 273-283.

Hu, C. and Yang, Y. (2010). An axiomatic characterization of a value for games in partition function form. SERIEs 1, 475-487.

Li F., Zhu Q., Liang L. (2018). Allocating fixed cost based on a DEA-game cross efficiency approach. Expert Systems with Applications 96, 196-207.

Macho-Stadler I., Pérez-Castrillo D., and Wettsein D. (2007). Sharing the surplus: an extension of the Shapley value for environments with externalities. Journal of Economic Theory 135, 339-356.

McQuillin B. (2009). The extended and generalized Shapley value: consideration of coalitional externalities and coalition structure. Journal of Economic Theory 144, 696-721.

Myerson R.B. (1977). Values for games in partition function form. International Journal of Game Theory 6, 23-31.

Parsons, S. and Wooldridge, M.(2002) Game theory and decision theory in multi-agent systems. Autonomous Agents and Multi-Agent Systems 5, 243-254

Pendharkar P.C. (2012). Game theoretical applications for multi-agent systems. Expert Systems with Applications 39, 273-279.

Sánchez-Pérez J.E. (2015). A note on a class of solutions for games with externalities generalizing the Shapley value. International Game Theory Review 17, 1-12.

Shapley L.S. (1953). A value for n-person games. In: Kuhn H.W., Tucker A.W. (eds.), Contributions to the theory of games II, Princeton University Press, New Jersey, pp. 307-317.

Skibski O., Michalak T.P., and Wooldridge M. (2018). The Stochastic Shapley value for coalitional games wit externalities. Games and Economic Behavior 108, 65-80.

Stanley R.P. (2011). Enumerative Combinatorics vol.1 (2nd edition). Cambridge Studies in Advanced Mathematics. Cambridge University Press.

Thrall, R. M. and Lucas, W. F. (1963). n-person games in partition function form. Naval Research Logistics 10, 281-298.

Torkaman A., Charcari N.M., Aghaeipour M. (2011). An approach for leukemia classification based on cooperative game theory. Analytical Celular Pathology 34, 235-246. 